\title{
TRASPLANTE RENAL EN PACIENTES CON ENTEROCISTOPLASTIA
}

\author{
J.M. ALAPONT ALACREU, J.J. PACHECO BRU, J.L. PONTONES MORENO, \\ M. ALONSO GORREA, J. SÁNCHEZ PLUMED, F.J. JIMÉNEZ CRUZ
}

Servicio de Urología. Hospital Universitario La Fe. Valencia.

Actas Urol Esp. 27 (4): 281-285, 2003

\section{RESUMEN}

"TRASPLANTE RENAL EN PACIENTES CON ENTEROCISTOPLASTIA"

OBJETIVOS: Evaluar la evolución y resultados del trasplante renal de donante cadáver en pacientes con ampliaciones vesicales realizadas por alteración del tracto urinario inferior (TUI).

PACIENTES Y MÉTODOS: Entre 1980 y 2001 hemos realizado 4 trasplantes renales en pacientes con fibrosis vesical restrictiva secundaria a tuberculosis ( 3 hombres y 1 mujer). Previamente se realizó ampliación vesical intestinal: 3 ileocecocistoplastias y 1 ileocistoplastia.

RESULTADOS: Tras 27 y 74 meses desde el trasplante renal 2 pacientes conservan buena función renal. Un paciente perdió el injerto por rechazo crónico a los 98 meses y el restante falleció a los 36 meses por sepsis de origen intestinal, conservando el injerto funcionante. Tres casos tuvieron complicaciones post-quirúrgicas que precisaron de tratamiento instrumental. Ningún paciente presentó incontinencia urinaria.

CONCLUSIONES: La enterocistoplastia es un procedimiento seguro y eficaz para pacientes con alteración del TUI y que precisan de trasplante renal.

PALABRAS CLAVE: Tuberculosis urinaria. Ampliación vesical. Trasplante renal.

\section{ABSTRACT}

"RENAL TRANSPLANTATION IN PATIENTS WITH AN AUGMENTATION CYSTOPLASTY"

OBJECTIVES: To asses the impact of augmentation enterocystoplasty on the success of cadaveric renal transplantation in patients with dysfunctional bladders.

PATIENTS AND METHODS: Between 1980 and 2001, 3 men and a woman with severe dysfunctional lower urinary tract underwent a total of 4 cadaveric renal transplantations. The etiologies of the bladder dysfunction were bladder contraction secondary to urinary tuberculosis in all cases. In 3 patients were performed an enterocystoplasty with ileocecal segment and one with ileon.

RESULTS: The overall allograft survival was 58.7 months. Two patients have functioning grafts 27 and 74 months after transplant, 1 has died due to an intestinal disease and other had crohnic rejection after follow-up of 98 months. Technical complications occurred in 3 patients. All patients remain continent without catheterization after the transplantation.

CONCLUSIONS: Enterocystoplasty is a safe and effective method of restoring lower urinary tract function in the patient with end stage renal disease and a small non compliant bladder.

KEY WORDS: Urinary tuberculosis. Augmentation enterocystoplasty. Renal transplant. 
$\mathrm{E}^{\mathrm{x}}$ xiste un número importante de pacientes con insuficiencia renal crónica (IRC) terminal debida a nefropatías por anomalías del tracto urinario inferior (TUI), bien de causa congénita (vejiga neurógena por mielomeningocele, enfermedad del cuello vesical, válvulas uretrales posteriores y estenosis de uretra) o adquirida (vejiga neurógena secundaria a traumatismos, enfermedades inflamatorias o degenerativas, fibrosis vesical por tuberculosis -TBC- o cistopatías intersticiales). Sin embargo la patología del TUI no supone hoy en día una contraindicación para el trasplante renal, aunque sí puede ser un serio condicionante del mismo. Presentamos nuestra experiencia en trasplante renal de pacientes con nefropatía secundaria a patología vesical restrictiva y realizamos una descripción de la literatura.

\section{PACIENTES Y MÉTODOS}

Entre febrero de 1980 y diciembre de 2001 hemos realizado en nuestro hospital un total de 1.326 trasplantes renales. De ellos 4 fueron en pacientes a los que previamente se les había realizado una ampliación vesical con intestino (3 hombres y una mujer). En todos los casos la alteración del TUI fue la fibrosis vesical por TBC, siendo ésta también la causa que motivó la IRC en 3 de ellos. El caso restante fue secundario a una nefropatía familiar. En la Tabla I observamos las edades de los pacientes, técnica quirúrgica y niveles plasmáticos de creatinina en el momento de realizar la ampliación vesical. En la ileocecocistoplastia aislamos el segmento ileocecal (aproximadamente $20 \mathrm{~cm}$ de íleon) el cual anastomosamos en su borde colónico a la vejiga trigonal, dejando el segmento ileal cerrado y referenciado a nivel de la fosa ilíaca derecha y de forma extraperitoneal, con la válvula ileocecal como mecanismo antirreflujo.

En 3 casos la neovejiga se realizó cuando los pacientes presentaban IRC, como paso previo a la inclusión en lista de espera para trasplante renal. En uno se hizo para evitar la IRC, aunque ésta apareció 14 años después. Las complicaciones post-quirúrgicas inmediatas (Tabla I) se resolvieron de forma conservadora: el hematoma retroperitoneal mediante punción percutánea y la trombosis de los troncos supra-aórticos (en un paciente afecto de disproteinemia familiar) con terapia anticoagulante. En los casos en los que la ampliación se había hecho como paso previo al trasplante se pautaron sondajes vesicales intermitentes con lavados de suero fisiológico para así mantener la distensibilidad vesical y eliminar las secreciones intestinales. La evolución hasta que se realizó el trasplante fue en general satisfactoria, con algunos episodios de infección urinaria en todos los pacientes. Un paciente que estaba en anuria presentó importantes molestias uretrales relacionadas con la expulsión de moco y que, pese al tratamiento pautado, no mejoraron hasta que se realizó el implante.

\section{RESULTADOS}

$\mathrm{El}$ injerto renal fue de donante cadáver en todos los casos, variando el tiempo en lista de espera hasta el trasplante entre 9 meses y 4 años (Tabla II). Con respecto a la técnica quirúrgica la anastomosis

\section{TABLA I}

CARACTERÍSTICAS PRINCIPALES DE LOS PACIENTES CUANDO SON SOMETIDOS A LA AMPLIACIÓN VESICAL Y EVOLUCIÓN POSTERIOR.

\begin{tabular}{|c|c|c|c|c|c|c|}
\hline Caso & $\begin{array}{l}\text { Edad } \\
\text { (años) }\end{array}$ & Motivo & Técnica & Complicaciones & $\begin{array}{l}\text { Creatinina } \\
(\mathbf{m g} / \mathbf{d l})\end{array}$ & Evolución \\
\hline 1 & 19 & TBC vesical & Ileocecocistoplastia & Ninguna & 1,2 & - Pielonefritis derecha \\
\hline 2 & 29 & $\begin{array}{l}\text { Preparación } \\
\text { trasplante }\end{array}$ & Ileocecocistoplastia & $\begin{array}{l}\text { Hematoma } \\
\text { retroperitoneal }\end{array}$ & 16 & $\begin{array}{l}\text { - ITU (gram-) } \\
\text { - Molestias uretrales (moco) }\end{array}$ \\
\hline 3 & 56 & $\begin{array}{l}\text { Preparación } \\
\text { trasplante }\end{array}$ & Ileocecocistoplastia & $\begin{array}{l}\text { Trombosis troncos } \\
\text { supra-aórticos }\end{array}$ & 12,2 & $\begin{array}{l}\text { - ITU (gram-) } \\
\text { - Insuficiencia cardiaca congestiva }\end{array}$ \\
\hline 4 & 21 & $\begin{array}{l}\text { Preparación } \\
\text { trasplante }\end{array}$ & Ileocistoplastia & Ninguna & 10,4 & - ITU (gram-) \\
\hline
\end{tabular}


TRASPLANTE RENAL Y EVOLUCIÓN POSTERIOR

\begin{tabular}{|c|c|c|c|c|c|c|c|}
\hline Caso & $\begin{array}{l}\text { Edad } \\
\text { (años) }\end{array}$ & $\begin{array}{l}\text { Tiempo } \\
\text { espera }\end{array}$ & Técnica & $\begin{array}{l}\text { Complicaciones y } \\
\text { tratamiento }\end{array}$ & $\begin{array}{l}\text { Creatinina } \\
\text { (1 mes después) }\end{array}$ & Urinocultivo + & Seguimiento \\
\hline 1 & 34 & 1 año & $\begin{array}{l}\text { Uréter a } \\
\text { asa ileal }\end{array}$ & $\begin{array}{l}\text { - Absceso perinjerto: } \\
\text { drenaje quirúrgico } \\
\text { - Oclusión intestinal: } \\
\text { tratamiento médico }\end{array}$ & $2,3 \mathrm{mg} / \mathrm{dl}$ & Ninguno & $\begin{array}{c}\text { Creatinina } \\
\text { sangre: } 2,4 \mathrm{mg} / \mathrm{dl} \\
(27 \text { meses })\end{array}$ \\
\hline 2 & 32 & 3 años & $\begin{array}{l}\text { Uréter a } \\
\text { asa ileal }\end{array}$ & $\begin{array}{l}\text { Urinoma: drenaje } \\
\text { percutáneo }\end{array}$ & $1,5 \mathrm{mg} / \mathrm{dl}$ & 6 (Pseudomonas) & $\begin{array}{l}\text { Rechazo crónico } \\
\text { (98 meses) }\end{array}$ \\
\hline 3 & 60 & 4 años & $\begin{array}{l}\text { Uréter a } \\
\text { asa ileal }\end{array}$ & $\begin{array}{l}\text { Fistula urinaria: } \\
\text { reimplante ureteral }\end{array}$ & $1,7 \mathrm{mg} / \mathrm{dl}$ & $\begin{array}{l}10 \text { (5 Pseudomonas, } 2 \\
\text { E. coli, resto: } \\
\text { Streptococcus, Serratia } \\
\text { y Klebsiella) }\end{array}$ & $\begin{array}{l}\text { Fallecido por } \\
\quad \text { causa } \\
\text { extraurológica } \\
\text { (36 meses) }\end{array}$ \\
\hline 4 & 21 & 9 meses & $\begin{array}{l}\text { Uréter a } \\
\text { vejiga } \\
\text { ileal }\end{array}$ & $\begin{array}{l}\text { Necrosis tubular } \\
\text { aguda: diálisis } 17 \text { días }\end{array}$ & $1,2 \mathrm{mg} / \mathrm{dl}$ & $\begin{array}{c}5 \text { (4 E coli y } 1 \\
\text { Streptococcus) }\end{array}$ & $\begin{array}{c}\text { Creatinina } \\
\text { sangre: } 1,6 \mathrm{mg} / \mathrm{dl} \\
\text { (74 meses) }\end{array}$ \\
\hline
\end{tabular}

arterial se hizo en 3 casos término-lateral a la ilíaca común y en 1 a la ilíaca externa, mientras que la venosa fue término-lateral a la ilíaca externa en todos. El uréter se anastomosó término-terminal al extremo del asa ileal en los casos de ileocecocistoplastia y espatulado sobre la neovejiga ileal en el restante. El post-operatorio inmediato cursó con complicaciones en todos los pacientes que se resolvieron con los tratamientos realizados (Tabla II). El caso 3 presentó una fístula urinaria secundaria a desinserción del uréter al asa ileal que precisó reimplantar el uréter a la neovejiga colónica, descubriéndose en esta cirugía una litiasis en el uréter proximal del injerto que conseguimos extraer mediante ureterolitotomía.

Todos los casos normalizaron los parámetros de función renal tras el trasplante (Tabla II). En la evolución posterior la complicación más frecuente ha sido la infección urinaria, principalmente por Pseudomonas y otros gram negativos (Tabla II), con buena respuesta a la antibioterapia pautada.

Tras 27 y 74 meses desde el trasplante 2 pacientes conservan buena función renal, uno perdió el injerto por rechazo crónico a los 98 meses y otro falleció a los 36 meses del trasplante por sepsis de origen intestinal, conservando el injerto funcionante.

\section{DISCUSIÓN}

En 1966 Kelly et al. ${ }^{1}$ describieron con resultados exitosos la ureteroileostomía cutánea como derivación urinaria para pacientes subsidiarios de trasplante renal y con TUI alterado. Hasta ese momento en estos pacientes estaba contraindicado el trasplante renal. Desde entonces han sido numerosas las publicaciones de trasplante renal en pacientes con derivación urinaria con resultados similares a los de Kelly et al. en este tipo de patología, tanto con conductos ileales como colónicos y ampliaciones vesicales ${ }^{2}$. La ureteroileostomía cutánea, dado sus buenos resultados en cistectomías, ha sido la técnica más usada en el pasado, sin embargo comporta un elevado número de complicaciones (estenosis del estoma, hiperqueratosis, infecciones urinarias, litiasis, contaminación micótica). Glass et al. ${ }^{3}$ tras revisar 68 pacientes con este tipo de derivación encuentran que existe un $32 \%$ de complicaciones atribuibles al conducto intestinal, por lo que concluyen que esta derivación debe ser evitada en estos pacientes. La rehabilitación vesical con autocateterismos e instilación de volúmenes crecientes de suero fisiológico tampoco muestra resultados positivos. La tendencia actual es a la recuperación de la funcionalidad de la vejiga siempre que sea posible. La enterocistoplastia, bien con colon o con íleon destubulizado, permite obtener vejigas de gran capacidad con presiones bajas a grandes volúmenes y sin riesgo de reflujo, lo que conlleva menor riesgo para el injerto. Además preserva la continencia urinaria, aumentando en gran medida la calidad de vida para el paciente. 
Todo esto ha llevado a que la enterocistoplastia sea en la actualidad el tratamiento de elección para pacientes que precisen de trasplante renal pero que tienen una vejiga pequeña por fibrosis.

La ampliación vesical puede ser realizada empleando distintos segmentos intestinales: estómago $^{4,5}$, íleon ${ }^{4-7}$, segmento ileo-cecal ${ }^{7,8}$ y sigma ${ }^{9}$. La tendencia es a usar segmentos destubulizados para así disminuir el riesgo de incontinencia urinaria. El uso de uno u otro depende en mayor grado de las preferencias y experiencia del cirujano que de los resultados de cada uno, aunque existen distintas complicaciones metabólicas y funcionales relacionadas con un segmento intestinal específico. El empleo de íleon y colon se ha asociado al desarrollo de acidosis metabólica hiperclorémica por absorción de amonio y cloruro que debe tratarse con alcalinizantes, sobre todo en niños para evitar problemas en el crecimiento. El empleo de estómago se asocia a alcalosis hipoclorémica por secreción de $\mathrm{HCl}$; debe tratarse con antagonistas de los receptores $\mathrm{H}_{2}$ o con inhibidores de la bomba de protones.

El uréter se anastomosa a la neovejiga con una técnica extravesical y preferentemente en la porción nativa de ésta ${ }^{10}$. Nosotros utilizamos el segmento ileocecal en 3 pacientes (los más antiguos) porque es relativamente fácil de realizar, tiene menor longitud de suturas anastomóticas que otras técnicas (mayor estanqueidad y menor riesgo de urinomas) y la anastomosis al íleon queda a nivel de fosa ilíaca derecha y extraperitoneal, con la válvula ileocecal como mecanismo antirreflujo. Tiene el inconveniente de que al no ser destubulizada las contracciones intestinales espontáneas podrían provocar incontinencia urinaria. Sin embargo en ninguno de los casos presentados ocurrió esta situación. La mayoría de autores prefiere realizar la ampliación como mínimo 3 meses antes del trasplante ${ }^{11}$ para que así los inmunosupresores no interfieran en el proceso de cicatrización. El inconveniente de esta opción es que en las neovejigas "secas" la colonización bacteriana crónica y la infección son muy difíciles de erradicar.

En estos pacientes, sobre todo si están en anuria, es muy importante realizar autocateterismos diarios e irrigaciones de la neovejiga con suero fisiológico para así mantener la distensibilidad de ésta y eliminar las secreciones intestinales.
En la serie de Hatch ${ }^{12}$ de 55 trasplantes renales en 50 pacientes con derivación intestinal la complicación más frecuente fue la infección urinaria, con una incidencia del $18 \%$ y una recurrencia del $10 \%$. Existen tres grupos de pacientes con un alto riesgo de infección urinaria y que deben beneficiarse de un seguimiento estricto y profilaxis antibiótica: los pacientes pediátricos, aquellos con rechazo agudo en tratamiento y pacientes que requieren de cistectomía y derivación después del trasplante. En las ampliaciones vesicales también son las infecciones de orina la complicación más frecuente pero no se ha demostrado que influya en la supervivencia del injerto ${ }^{6}$. En nuestra serie todos los pacientes menos 1 han tenido infecciones urinarias de repetición, con una supervivencia media del implante renal de 58,7 meses.

Existe un riesgo potencial, acentuado al ser pacientes inmunodeprimidos, de desarrollar un tumor en el segmento intestinal ${ }^{13,14}$ por lo que el seguimiento con cistoscopia anual parece justificado de por vida.

Coincidimos con la mayoría de autores en que, y pese al bajo número de casos publicados, el trasplante renal en vejigas ampliadas es un procedimiento seguro y eficaz. La morbilidad post-quirúrgica en nuestra serie ha sido elevada, sin embargo la evolución posterior ha sido satisfactoria, tanto para el paciente como para el implante, con una supervivencia del mismo comparable al resto de trasplantados. Por el elevado riesgo de complicaciones, sobre todo infecciones, es muy importante el control riguroso de estos pacientes.

\section{REFERENCIAS}

1. KELLY WD, MERKERL FK, MARKLAND C.: Ileal urinary diversion in conjunction with renal homotransplantation. Lancet 1966; 1: 222-226.

2. NGUYEN DH, REINBERG Y, GONZÁLEZ R, FRYD D, NAJARIAN JS.: Outcome of renal transplantation after urinary diversion and enterocystoplasty: a retrospective, controlled study. J Urol 1990; 144: 1349-1351.

3. GLASS NR, UEHLING D, SOLLINGER H, BELZER F.: Renal transplantation using ileal conduits in 5 cases. J Urol 1985; 133: 666-668.

4. ALFREY EJ, CONLEY SB, TANNEY DC et al.: Use of an augmented urinary bladder can be catastrophic in renal transplantation. Transplant Proc 1997; 29: 154155. 
5. BURNS MW, WATKINS SL, MITCHELL ME, TAPPER D.: Treatment of the bladder dysfunction in children with end-stage renal disease. J Pediatr Surg 1992; 27: 170-174.

6. RISCHMANN P, MALAVAUD B, BITKER MO et al.: Results of 51 renal transplants with the use of bowel conduits in patients impaired bladder function: a retrospective multicenter study. Transplant Proc 1995; 27: 2427-2429.

7. NAHAS WC, MAZZUCCHI E, ANTONOPOULOS I et al.: Kidney transplantation in patients with bladder augmentation: surgical outcome and urodynamic follow-up. Transplant Proc 1997; 29: 157-158.

8. BARNETT MG, BRUSKEWITZ RC, BELZER FO, SOLLINGER HW, UEHLING DT.: Ileocecocystoplasty bladder augmentation and renal transplantation. $J$ Urol 1987; 138: 855-858.

9. LÓPEZ-PEREIRO P, LÓPEZ-GUTIÉRREZ JC, JAUREGUIZAR E.: Desderivación, colocistoplastia y trasplante renal. Arch Esp Urol 1990; 43: 983-985.

10. SHELDON CA, GONZÁLEZ R, BURNS MW, GILBERT A, BUSON H, MITCHELL ME.: Renal transplantation into the dysfunctional bladder: the role of adjunctive bladder reconstruction. J Urol 1994; 152: 972-975.
11. GONZÁLEZ-MARTÍN M, NOVAS-CASTRO S, ÁLVAREZ-CASTELO L, CHANTADA-ABAL V, SÁNCHEZRODRÍGUEZ J, DUARTE-NOVO J.: Enterocystoplasty and renal transplantation. J Urol 2001; 165: 393-396.

12. HATCH DA, BELITSKY P, BARRY JM et al.: Fate of renal allografts transplanted in patients with urinary diversion. Transplantation 1993; 56: 838-842.

13. FILMER RB, SPENCER JR.: Malignancies in bladder augmentations and intestinal conduits. J Urol 1990; 143: 671-678.

14. SHOKEIR AA, SHAMAA M, EL-MEHRESH MM, ELBAZ M, GONEIM MA.: Late malignancy in bowel segments exposed to urine without faecal stream. Urology 1995; 46: 657-661.

Dr. J.M. Alapont Alacreu

Servicio de Urología

Hospital Universitario La Fe

Avda. Campanar, 21

46009 Valencia

(Trabajo recibido el 15 octubre de 2002) 